\title{
Mechanical Trapping in a Quadratically Coupled Optomechanical Double Disk
}

\author{
Jeffrey T. Hill, Qiang Lin, Jessie Rosenberg, and Oskar Painter \\ Thomas J. Watson, Sr., Laboratories of Applied Physics, California Institute of Technology, Pasadena, CA \\ 91125, USA \\ jthill@caltech.edu
}

\begin{abstract}
We demonstrate a novel approach for trapping mechanical motion and elastic tuning of mechanical rigidity while preserving phonon number, through a giant quadratic optomechanical coupling created from a tunable coupled microdisk resonator.

(c) 2011 Optical Society of America

OCIS codes: (220.4880) Optomechanics; (270.0270) Quantum optics
\end{abstract}

Cavity optomechanics has attracted significant attention for the intriguing manipulation of mesoscopic mechanical motion. Although most of the current work focuses on the linear optomechanical coupling aiming for controlling motion via mechanical amplification/cooling [1], recently there has been increasing interest in quadratic optomechanical $\left(x^{2}\right)$ coupling with the potential for probing phonon number in the non-demolition fashion [2]. However, $x^{2}$ coupling exhibits physics far more profound than the quantum non-demolition measurement itself [3]. Here we report a novel approach for simultaneously trapping both the optical resonance and mechanical motion, based upon an extremely strong $x^{2}$ coupling about six orders of magnitude larger than any other systems. Such an approach is able to manipulate the mechanical rigidity in a purely elastic fashion preserving the phonon number, in strong contrast to the conventional optical spring which is inevitably accompanied with mechanical-energy perturbation by dynamic backaction. Our demonstrated scheme is able to control the mechanical rigidity nearly instantaneously within the cavity photon lifetime, thus opening the application potential for optomechanical signal processing with an ultrafast response.
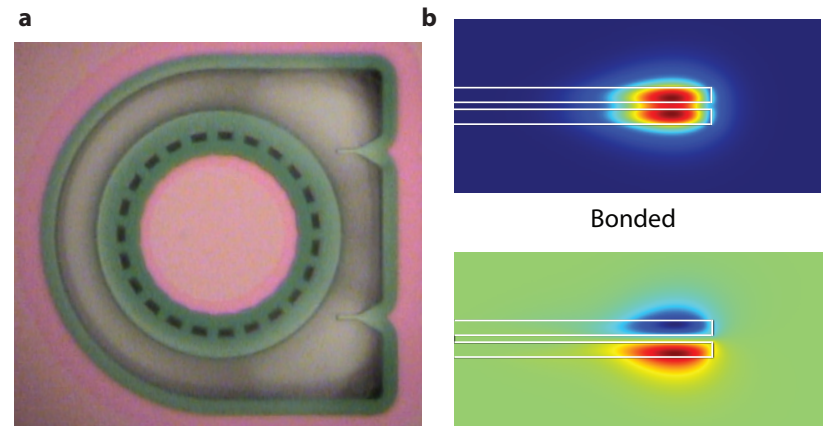

Anti-bonded

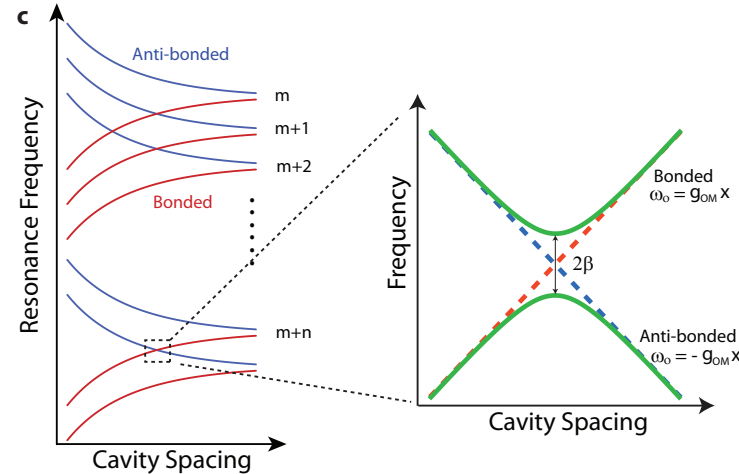

Fig. 1. (a) Optical micrograph of a double disk resonator with a diameter of $60 \mu \mathrm{m}$. (b) Mode profiles of bonded and antibonded modes. (c) Schematic of bonded and anti-bonded mode tuning creating an anti-crossing.

Our proposed scheme is based on a coupled microdisk resonator composed of two stacked 400-nm thick silica microring resonators separated by a 150-nm inner amorphous silicon support pilar (Fig. 1a,b). In contrast to our previous devices [4], we engineer the slot pattern for desired mechanical flexibility (Fig. 1a). The small separation between the two disks leads to strong optical coupling and split the cavity modes into bonded (symmetric) and antibonded (anti-symmetric) supermodes (Fig. 1b), whose frequencies depend sensitively, but oppositely, on the disk spacing. Interestingly, the whispering-gallery nature of the device supports many optical modes separated by freespectral range. As a result, changing the disk spacing allows us to cross in frequency the bonded and anti-bonded modes from different mode families by tuning them in opposite directions (Fig. 1c). By inducing the coupling between these coincident modes, an anti-crossing can be created where the cavity resonance depends quadratically on the mechanical displacement, $\omega_{0} \propto g^{\prime} x^{2} / 2$, with the quadratic coupling coefficient $g^{\prime}=g_{O M}^{2} / \beta$ where $\beta$ is half of the mode splitting at the anti-crossing and $g_{O M}$ is the linear optomechanic coupling of the modes (Fig. 1c). Clearly, a large quadratic coupling can be produced with a large $g_{O M}$ and a small $\beta$. We engineer the magnitude of the mode coupling, $\beta$, by varying the disk's sidewall angle to break the mirror symmetry of the coupled cavities, resulting in a small mode splitting in the order of cavity linewidth $\left(\sim 1 \mathrm{pm}\right.$ in the 980 -nm band). With a $g_{O M} / 2 \pi=60 \mathrm{GHz} / \mathrm{nm}$ among the largest reported so far [5], we are able to realize an extremely large quadratic coupling. Figure 2a shows an example. Fitting 


\section{CThJ3.pdf}

the quadratic optomechanical dependence results in $g^{\prime} / 2 \pi=5.8 \mathrm{THz} / \mathrm{nm}^{2}$, which is about 6 orders of magnitude larger than those in any other system [2].

The pure quadratic nature of optomechanical coupling at the anti-crossing region is shown clearly in Fig. $2 \mathrm{~b}$ where the linear transduction of mechanical motion is dramatically suppressed by about $43 \mathrm{~dB}$ when we move the laser frequency from the linear coupling region into the anti-crossing point. Apart from non-demolition probing of phonon number, such a quadratic coupling exhibits another intriguing feature capable of producing a mechanical potential for trapping the mechanical motion, since the optical force exerted by a photon is given by $f=-d \omega_{0} / d x=-\hbar g^{\prime} x$, leading to a per-photon spring of $\hbar g^{\prime}$. Note that this $x^{2}$-coupling-induced spring is purely elastic in nature and thus provides an excellent approach to engineer the mechanical rigidity, since it does not have any effect on the total mechanical energy, thus preserving the phonon number. This novel type of static optically induced spring is quite distinct from the optical spring induced by the dynamic back-action resulting from linear optomechanical coupling, which inevitably perturbs the energy of the mechanical mode and also relies critically on the laser-cavity detuning. In contrast, this static optical spring depends only on the intra-cavity photon number. For a given input power to the cavity, $P_{i}$, the total spring induced by this quadratic coupling is $\delta k= \pm g^{\prime} P_{i} /\left(\omega_{0} \Gamma_{0}\right)$ where the sign depends on whether the upper or lower branch mode is used. This static optical spring is also quite distinct from the mechanical trapping proposed in Ref. [6] which still relies on cavity resonance shape and thus affected by dynamic back-action.
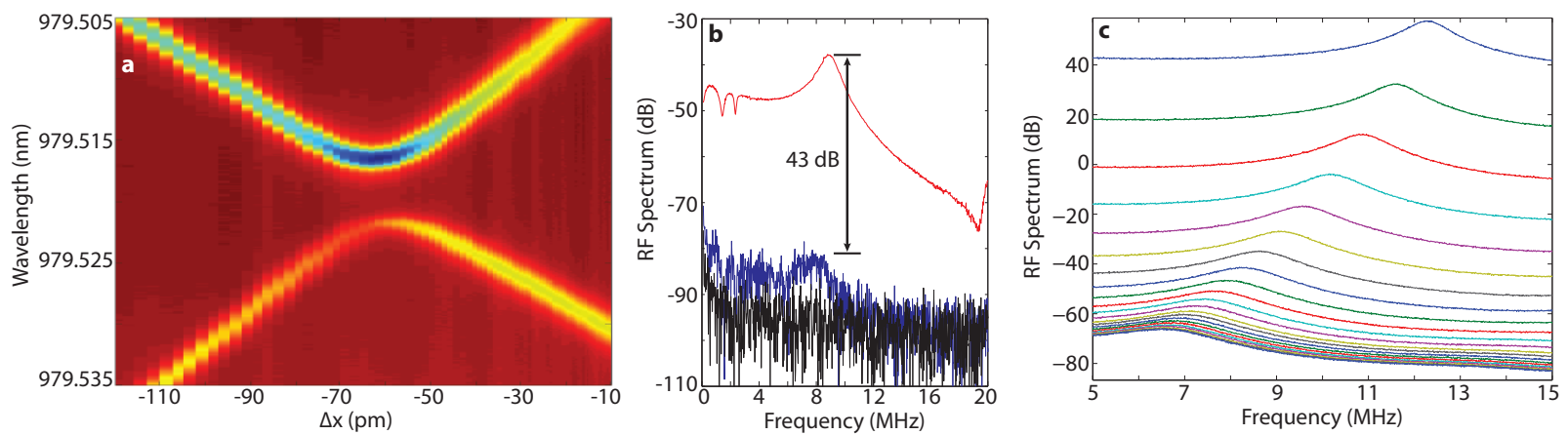

Fig. 2. (a) Bonded and anti-bonded modes tuned through the anti-crossing. (b) RF spectrum showing $43 \mathrm{~dB}$ of suppression of the linear transduction (red) at the anti-crossing (blue) relative to the noise floor (black). (c) RF spectra showing increasing mechanical frequency with input power (curves bottom to top vertically displaced proportional to input power).

For the strong quadratic coupling in our device, each photon inside the cavity is able to produce a spring constant of $4 \mathrm{mN} / \mathrm{m}$. Therefore, even a moderate power launched into the cavity is able to efficiently trap the mechanical motion and to dramatically modify the mechanical rigidity. This is shown in Fig. 2c. The mechanical mode of our device has an intrinsic spring of $133 \mathrm{~N} / \mathrm{m}$ with an intrinsic mechanical frequency of $3.7 \mathrm{MHz}$. It exhibits a mechanical frequency of $\sim 6.5 \mathrm{MHz}$ in $1 \mathrm{~atm}$ of $N_{2}$ environment resulting from squeeze-film damping. However, by launching only 221 $\mu \mathrm{W}$ power to the cavity mode, we are able to push the mechanical frequency up to $\sim 12.5 \mathrm{MHz}$, implying an increase of mechanical rigidity by more than one order of magnitude compared with the intrinsic mechanical rigidity. Further increase can be easily realized by putting more photons into the cavity.

Such an elastic modification of mechanical rigidity implies a significant improvement of mechanical quality which can be measured in a vacuum environment. Moreover, thanks to its elastic nature, such a spring can be applied by the optical wave nearly instantaneously within the cavity photon lifetime. Consequently, our demonstrated optomechanical trapping and elastic tuning of mechanical rigidity thus open a great potential for various applications such as flexible switching between good- and band-cavity regime, ultrafast optomechanical signal processing, efficient classical and quantum squeezing of mechanical motion, etc, which will be explored in the near future.

\section{References}

1. T. J. Kippenberg and K. J. Vahala, "Cavity Optomechanics: Back-Action at the Mesoscale," Science 321, 1172 (2008).

2. J. D. Thompson, et al, "Strong dispersive coupling of a high-finesse cavity to a micromechanical membrane," Nature 452, 72 (2008).

3. G. Heinrich, et al, "Photon shuttle: Landau-Zener-Stuckelberg dynamics in an optomechanical system," Phys. Rev. A bf 81, 011801(R) (2010).

4. J. Rosenberg, et al, "Static and dynamic wavelength routing via the gradient optical force," Nature Photon. 3, 478 (2009).

5. D. Van Thourhout and J. Roels, “Optomechanical device actuation through the optical gradient force," Nature Photon. 4, 211 (2010).

6. P. T. Rakich, et al, "Trapping, corralling and spectral bonding of optical resonances through optically induced potentials," Nature Photon. 1, 658 (2007). 